\title{
Efficacy of Silicone Gel Sheets with 595-nm Pulse Dye Laser in Patients with Post-Cesarean Section Scar: A Pilot Study
}

Keywords: Cesarean section; Postoperative scar; Scar revision; Silicone gel sheet

\begin{abstract}
Background: The cesarean section site is the lower abdomen, where postoperative scarring can cause patient distress. There is no genera agreement about the best option for therapeutic management of cesarean section scars.

Objective: The aim of this study was to evaluate the efficacy and safety of the silicone gel sheet for cesarean section scars.

Methods: Eight volunteers (Korean women) who had undergone cesarean section on the lower abdomen were enrolled. This study involved an open-label, split-scar trial. First, the 595-nm pulsed dye laser ( $1.5 \mathrm{~ms}, 7 \mathrm{~mm}$ spot size, $7.5-15 \mathrm{~J} / \mathrm{cm} 2,1$ session) was performed on all the cesarean scars. Silicone gel (ScarclinicTM) was applied on the right half (case side) for 24 hours each day for 12 weeks and not on the other half (control side). Clinical assessment was carried out using a Vancouver scar scale (VSS). The investigator's global assessments of the final cosmetic results were rated.

Results: The mean difference in the VSS score from baseline to one month was $2.4(p=0.01)$ on the case side and $0.4(p=0.08)$ on the control side. The mean difference in the VSS score from baseline to three months was $2.7(p=0.03)$ on the case side and $0.7(p=0.17)$ on the control side. The average global assessment scores on the case side were $2.4 \pm 0.5$ after one month and $2.5 \pm 0.5$ after three months. The average global assessment scores on the control side were 2.1 \pm 0.4 after one month and $2.3 \pm 0.5$ after three months. There were no significant adverse effects such as pain, hyper pigmentation, or infection.
\end{abstract}

Conclusion: The treatment of postoperative cesarean section scars using a silicone gel sheet may be effective and safe.

\section{Introduction}

Postoperative scarring is one of the most common concerns among surgical patients. Cesarean section refers to the delivery of a baby through surgical incisions in the abdomen and uterus. The Cesarean section site is the lower abdomen, where postoperative scarring can cause patient distress. There is no general agreement about the best option for therapeutic management of cesarean section scars. Intralesional steroids, cryosurgery, pressure therapy, dermabrasion, 5-fluorouracil, silicone gel sheet therapy, laser therapy, and excisional surgery continue to be used and debated [1].

Silicone gel sheet therapy has the advantages of few systemic side effects, cost-effectiveness, and safety as compared with excision or laser therapy. It also induces no pain, and therefore could be applied to children's scars. There are also reports of the preventive and improvement effects of these sheets on hypertrophic and keloid

\section{Journal of}

\section{Clinical \& Investigative} Dermatology

Joon Hyuk Suh ${ }^{1}$, Sun Young Choi ${ }^{1}$, Tae Rin Kwon ${ }^{2}$, Song $\mathrm{I} \mathrm{Im}^{2}$ and Beom Joon $\mathrm{Kim}^{1 *}$

'Department of Dermatology, Chung-Ang University College of Medicine, Seoul, South Korea

${ }^{2}$ Department of Biomedical Science, College of Medicine, Chung-Ang University, Seoul, South Korea

\section{*Address for Correspondence}

Beom Joon Kim, Department of Dermatology, Chung-Ang University Hospital, 102 Heukseok-ro, Dongjak-Gu, Seoul 156-755, South Korea, Tel: 82-2-6299-1525; Fax: 82-2-823-1049; E-mail: beomjoon@unitel.co.kr

Submission: 10 September 2015

Accepted: 13 November 2015

Published: 17 November 2015

Copyright: ( 2015 Suh JH, et al. This is an open access article distributed under the Creative Commons Attribution License, which permits unrestricted use, distribution, and reproduction in any medium, provided the original work is properly cited.

scars [2-4], and some of studies report that the sheet was significantly effective in reducing postoperative incision wound scarring $[5,6]$. The present prospective study was designed to evaluate the efficacy and safety of the silicone gel sheet for use with cesarean section scars.

\section{Materials and Methods}

Eight volunteers (Korean women) were enrolled. All patients who had cesarean section on the lower abdomen were selected between January of 2005 and December of 2014. Participants were excluded if they reported a history of keloid scars, active cutaneous inflammation, or current pregnancy or had been undergoing any treatment with topical agents or laser therapy before the study.

This study involved an open-label, split-scar trial. First, The 595$\mathrm{nm}$ pulsed dye laser (V-beam, Candela Corp, Wayland, MA, US), tuned to $595-\mathrm{nm}, 1.5 \mathrm{~ms}$ pulse duration, $7 \mathrm{~mm}$ spot size and a fluence of $7.5-15 \mathrm{~J} / \mathrm{cm}^{2}$, was performed on all of the cesarean scars on each of the participants at day 0 (each patient was treated for one session using the same parameters). In some patients, local anesthesia with a topical lidocaine-prilocaine mixture (EMLA cream; AstraZeneca, Luton, U.K.) was used depending on preference. The scar was divided equally into two parts. Silicone gel was applied on the right half (Scarclinic ${ }^{\mathrm{TM}}$, Ildong Pharmaceutical Co, Ltd. Seoul, South Korea) and not on the other half. The silicone gel sheet was tailored to half the size of the scar and was placed over the scar. The patients used the silicone gel sheet for 24 hours each day for 12 weeks.

All of the patients were followed up at baseline, 4 weeks, and 12 weeks. The same photographer photographed the participants using identical camera settings and lighting conditions at every visit (Canon EOS40D, 10.0 megapixels, Tokyo, Japan). Clinical assessment was carried out using a Vancouver scar scale (VSS) that measured pigmentation $(0=$ normal, $1=$ hypopigmentation, $2=$ mixed 
Citation: Kim BJ, Suh JH, Choi SY, Kwon TR, Song II. Efficacy of Silicone Gel Sheets with 595-nm Pulse Dye Laser in Patients with Post-Cesarean Section Scar: A Pilot Study. J Clin Investigat Dermatol. 2015;3(2): 4.

pigmentation, 3 = hyperpigmentation), pliability $(0=$ normal, $1=$ supple, $2=$ yielding, $3=$ firm, $4=$ ropes, $5=$ contracture), vascularity $(0=$ normal, $1=$ pink, $2=$ red, $3=$ purple $)$, and height $(0=$ flat, 1 $\leq 2 \mathrm{~mm}, 2=2-5 \mathrm{~mm}, 3 \geq 5 \mathrm{~mm}$ ) [7]. Two independent physicians also interpreted the results. The score for each parameter was assessed separately, and then all four scores were summed. The score for each parameter was assessed separately, and then all four scores were summed. Two independent investigator's global assessments of final cosmetic results were rated as poor (1), fair (2), good (3), or excellent (4). Statistical analyses were performed using SPSS version 18.0 for Windows (SPSS Inc., Chicago, IL). Results are expressed as mean \pm standard deviation (SD). A paired t-test was performed in order to evaluate the mean VSS difference between two groups. P-values less than 0.05 were considered statistically significant.

\section{Results}

There were eight women with a mean age of $37 \pm 2.5$ years (range 34-41 years). The mean number of cesarean sections was $1.5 \pm 0.5$ (range 1-2) (Table 1). The mean time from the latest incision to the start of treatment with silicone gel sheets was $4.6 \pm 3.1$ years (range 1-9 years) (Table 2 ).

The mean VSS scores on both sides were $7.0 \pm 3.8$ before the treatment, $4.6 \pm 2.9$ one month after treatment, and $4.3 \pm 2.5$ three months after treatment with one session of 595-nm pulsed dye laser (PDL) and application of the silicone-gel sheet (case). Meanwhile, scores were $6.6 \pm 3.3$ one month after treatment and $6.5 \pm 2.9$ three months after treatment with only one session of 595-nm pulsed dye laser (PDL) (control). The mean VSS difference from baseline to one month was $2.4(p=0.01)$ on the case side and $0.4(p=0.08)$ on the control side. The mean VSS difference from baseline to three months was $2.7(p=0.03)$ on the case side and $0.7(p=0.17)$ on the control side (Figure 1 and Table 3 ). The mean VSS differences were significantly decreased on the case side (Figure 2). The average investigator's global assessment scores on the case side were $2.4 \pm 0.5$ after one month and

Table 1: Demography and baseline characteristics.

\begin{tabular}{|c|c|c|}
\hline Patient & Sex/Age & Number of cesarean section \\
\hline 1 & $F / 41$ & 2 \\
\hline 2 & $F / 40$ & 1 \\
\hline 3 & $F / 39$ & 1 \\
\hline 4 & $F / 36$ & 2 \\
\hline 5 & $F / 36$ & 1 \\
\hline 6 & $F / 37$ & 2 \\
\hline 7 & $F / 34$ & 1 \\
\hline 8 & $F / 40$ & 2 \\
\hline
\end{tabular}

Table 2: Interval from cesarean section to silicone gel sheet use and the prior treatment.

\begin{tabular}{|c|c|}
\hline Parameter & No. \\
\hline \begin{tabular}{c|} 
Interval from the latest incision to treatment with \\
silicone gel sheet (months)
\end{tabular} \\
$\leq 12$ & 2 \\
$13-24$ & 0 \\
$25-36$ & 1 \\
$\geq 37$ & 5 \\
\hline
\end{tabular}

Table 3: Investigators' average clinical scar assessment (VSS score) after treatment with one session of 595-nm Pulsed-Dye Laser and applying silicone gel sheets for 12 weeks. ( $\left.{ }^{*} p<0.05\right)$

\begin{tabular}{|c|l|c|c|c|}
\hline \multirow{2}{*}{ Case } & Average & $\begin{array}{c}\text { Standard } \\
\text { deviation }\end{array}$ & $p$-value \\
\cline { 2 - 5 } & Baseline - 4wks & 2.375 & 1.302 & 0.001 \\
\cline { 2 - 5 } & Baseline - 12wks & 2.750 & 1.753 & 0.003 \\
\hline \multirow{2}{*}{ Control } & Baseline - 4wks & 0.375 & 0.518 & 0.080 \\
\cline { 2 - 5 } & Baseline - 12wks & 0.500 & 0.926 & 0.170 \\
\hline
\end{tabular}

A paired t-test was performed. $P$-values less than 0.05 were considered statistically significant.

Table 4: Average investigators' global assessments after treatment with one session of 595-nm Pulsed-Dye Laser and applying silicone gel sheets.

\begin{tabular}{|c|c|c|}
\hline \multirow{2}{*}{} & \multicolumn{2}{|c|}{ Mean investigators' global assessment } \\
\cline { 2 - 3 } 4 weeks & Control (PDL only) & Case (PDL + silicone-gel) \\
\hline 12 weeks & $2.1 \pm 0.4$ & $2.4 \pm 0.5$ \\
\hline
\end{tabular}

Investigators' global assessment scale: poor (1), fair (2), good (3), or excellent (4).

$2.5 \pm 0.5$ after three months. The average global assessment scores on the other side were $2.1 \pm 0.4$ after one month and $2.3 \pm 0.5$ after three months (Table 4). There were no significant adverse effects such as pain, hyper pigmentation, or infection.

\section{Discussion}

The postsurgical wound healing process is associated with various cellular activities and lasts for several months. Because a number of factors, including the patient's age, sex, underlying disease, cutaneous constitution, tension of suture site, and movement of the muscles, can affect the wound healing process, scars can inevitably be raised or widened.

To minimize scar formation during the healing process, several treatment options have been introduced such as intralesional corticosteroids, filler injection, cryosurgery, radiotherapy, pressure therapy, dermabrasion, 5-fluorouracil, bleomycin, excisional surgery, laser peeling, and silicone gel application. These methods have shown varying results; therefore, there is no standard treatment for scar revision. Additionally, once scars have matured, it is difficult to recover completely to the previous state. Therefore, the efficacy or usefulness of these numerous interventions remains arguable.

Pulsed-dye laser (PDL) is one of the laser therapies for scar revision. PDL selectively targets hemoglobin and coagulates the microvasculature in the capillary and reticular dermis, resulting in the destruction of pathologic neovascularization, by the principle of selective photothermolysis. Scars become flattening, depigmentation, normalization of surface irregularity and improvement of the texture by damaging the vessels [8]. Kuo et al. reported on the reduction of transforming growth factor- $\beta 1$ expression, fibroblast proliferation, and collagen type II deposition, an upregulation of matrix metalloproteinase and keloid fibroblast apoptosis following PDL treatment [9].

The application of silicone gel, one of the interventions for scar revision, has also been suggested to prevent scar formation during the postoperative period. The mechanism of action of silicone gel sheets is not fully explained. Accordingly, several postulations 


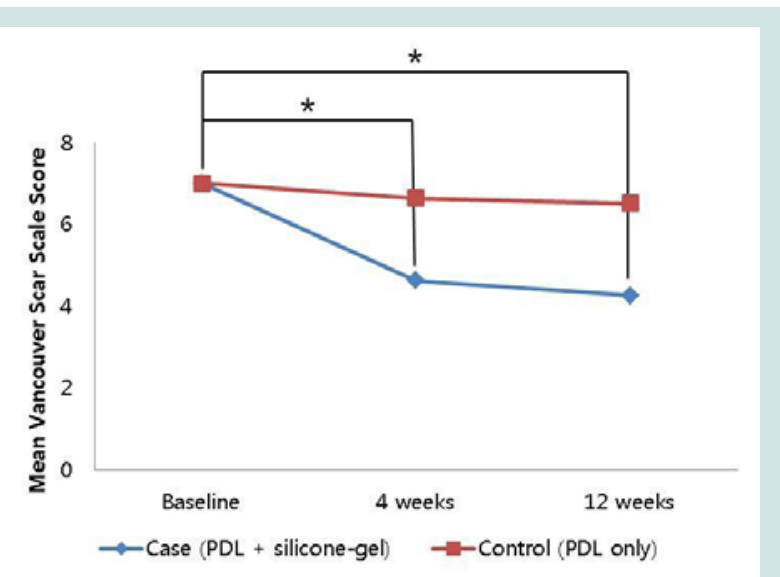

Figure 1: Investigators' average clinical scar assessment (VSS score) after treatment with one session of 595-nm Pulsed-Dye Laser and applying silicone gel sheets for 12 weeks. $\left({ }^{*} p<0.05\right)$

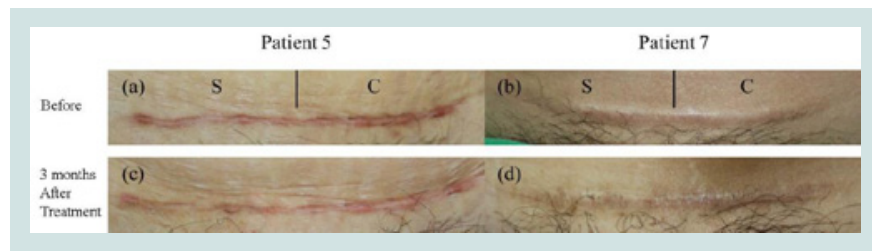

Figure 2: Clinical results of silicone gel sheet application on half of the scar. The photographs on the upper $(a, b)$ were taken before treatment, and those on the lower (c, d) were taken three months after the treatment. S: Applying silicone gel sheet side, C: Control side.

were suggested to understand the efficacy of silicone gel in treating scars. These hypotheses included increased temperature or oxygen tension, polarization of scar tissue caused by negative static charge, and modulation of growth factors [10,11]. Meanwhile, it has been proposed that some signal substances produced in the moist environment under the gel sheet regulate activity of keratinocytes on fibroblasts. Davey et al. explained that the relatively impermeable silicone gel acts in the same way as the stratum corneum [12]. Mustoe reported the mechanism that the silicone gel reduces water vapor loss and restores homeostasis to the scar, thereby reducing capillary hyperemia, collagen deposition, and hypertrophic scar formation [13].

In this study, we used VSS scores to provide an objective measurement of scarring. First, we performed one session of PDL on both side at baseline. The effect of scar revision by PDL was identified previous studies. Through performing the PDL at baseline, we expected the improvement of the all scar lesions, combination effect with silicone sheet. And then patients were put on a silicone sheet the scar lesions. Mean VSS scores for the silicone gel side of the cesarean section scars were statistically decreased one and three months after treatment $(p<0.05)$. The result might reflect the effect of treatment, which combined PDL and silicone in post-cesarean section scars. Meanwhile, mean VSS score for the other side, on which was no silicone-gel sheet was applied, were not statistically decreased. It is thought that the effect of greater scar improvement on the silicone gel side was caused by the silicone gel rather than the PDL. The mean global assessment score on the silicone-gel sheet side was 2.5 three months after treatment. The global assessment also indicated better cosmetic outcomes. Common adverse effects resulting from laser irradiation or gel application, including pigment change, delayed wound healing, or severe pain, did not occur in this study.

5 subjects went through the scar treatment more than 3 years after the incision. Mean VSS differences (baseline- 12 weeks), was higher in fresh scars than old scars. However, there was no statistically significant. Therefore, we thought that the intervention of PDL and silicone also could be effective in old scars. This study was followed up to 12 weeks. It was hard to evaluate the long-term effect of the intervention. Therefore, further studies, which recruit more people and perform the randomized controlled trial, are needed to determine the long-term efficacy and safety.

We found improvement in post-cesarean section scars after treatment with one session of PDL and application of silicone gel. Although we did not indicate more than three months of followup data, our findings suggest that scar management using siliconegel sheets may relieve scar formation and aid in roe rapid scar improvement than with no treatment. A second limitation is that the number of participants was small and consisted only of Korean patients. It is thought to be prudent to enroll more cesarean section women, especially those who underwent cesarean section recently. An additional limitation of this study is that there was no histologic assessment of treated skin. Determining collagen and elastin differences between each side would better help us in understanding the evolution of these treated scars. Finally, when applying the silicone gel sheet, some patients who used bandages or tape to prevent detaching the sheet arbitrarily may have influenced the scar pigmentation.

Based on this pilot study, the treatment of postoperative scarring using a silicone gel sheet is safe and effective. Scar management using a silicone gel sheet may improve the quality of life of patients with surgical scarring. Further studies are needed to evaluate the effect of the treatment on other scars, such as traumatic and burn scars. It is also necessary to evaluate scars at other sites, such as facial scars and thyroidectomy scars on the neck. Furthermore, long-term follow-up is needed to compare the course of scarring of treated and untreated scars using this material.

\section{References}

1. Alster TS, Tanzi EL (2003) Hypertrophic scars and keloids: etiology and management. Am J Clin Dermatol 4: 235-243.

2. Sproat JE, Dalcin A, Weitauer N, Roberts RS (1992) Hypertrophic sternal scars: silicone gel sheet versus Kenalog injection treatment. Plast Reconstr Surg 90: 988-992.

3. Perkins K, Davey RB, Wallis KA (1983) Silicone gel: a new treatment for burn scars and contractures. Burns Incl Therm Inj 9: 201-204.

4. Quinn KJ (1987) Silicone gel in scar treatment. Burns Incl Therm Inj 13 Suppl: S33-S40.

5. Mustoe TA (2008) Evolution of silicone therapy and mechanism of action in scar management. Aesthetic Plast Surg 32: 82-92.

6. Signorini M, Clementoni MT (2007) Clinical evaluation of a new self-drying silicone gel in the treatment of scars: a preliminary report. Aesthetic Plast Surg 31: 183-187.

7. Sullivan T, Smith J, Kermode J, Mclver E, Courtemanche DJ (1990) Rating the burn scar. J Burn Care Rehabil 11: 256-260. 
Citation: Kim BJ, Suh JH, Choi SY, Kwon TR, Song II. Efficacy of Silicone Gel Sheets with 595-nm Pulse Dye Laser in Patients with Post-Cesarean Section Scar: A Pilot Study. J Clin Investigat Dermatol. 2015;3(2): 4.

ISSN: 2373-1044

8. Taniguchi Y, Muraoka M, Harada T, Ito N (2003) Pulsed dye laser treatment for linear scars with concave and erythematous appearance. Aesthetic Plast Surg 27: 205-208.

9. Kuo YR, Jeng SF, Wang FS, Chen TH, Huang HC, et al. (2004) Flashlamp pulsed dye laser (PDL) suppression of keloid proliferation through downregulation of TGF-beta1 expression and extracellular matrix expression Lasers Surg Med 34: 104-108.

10. Ko WJ, Na YC, Suh BS, Kim HA, Heo WH, et al. (2013) The effects of topical agent (kelo-cote or contractubex) massage on the thickness of post-burn scar tissue formed in rats. Arch Plast Surg 40: 697-704.

11. Shih R, Waltzman J, Evans GR; Plastic Surgery Educational Foundation Technology Assessment Committee (2007) Review of over-the-counter topical scar treatment products. Plast Reconstr Surg 119: 1091-1095.

12. Davey RB, Wallis KA, Bowering K (1991) Adhesive contact media-- an update on graft fixation and burn scar management. Burns 17: 313-319.

13. Mustoe TA (2008) Evolution of silicone therapy and mechanism of action in scar management. Anesthetic Plast Surg 32: 82-92. 